\title{
Multiscale Fractal Descriptors Applied to Nanoscale Images
}

\author{
João B. Florindo ${ }^{1}$ \\ Mariana S. Sikora ${ }^{2}$ \\ Ernesto C. Pereira ${ }^{2}$ \\ Odemir M. Bruno ${ }^{1}$ \\ ${ }^{1}$ Physics Institute of São Carlos, University of São Paulo \\ São Carlos, SP, Brazil \\ jbflorindo@ursa.ifsc.usp.br, bruno@ifsc.usp.br \\ ${ }^{2}$ Interdisciplinary Electrochemistry and Ceramics Laboratory \\ Federal University of São Carlos, São Carlos, SP, Brazil \\ ma_sikora@hotmail.com,decp@power.ufscar.br
}

\begin{abstract}
This work proposes the application of fractal descriptors to the analysis of nanoscale materials under different experimental conditions. We obtain descriptors for images from the sample applying a multiscale transform to the calculation of fractal dimension of a surface map of such image. Particularly, we have used the Bouligand-Minkowski fractal dimension. We applied these descriptors to discriminate between two titanium oxide films prepared under different experimental conditions. Results demonstrate the discrimination power of proposed descriptors in such kind of application.
\end{abstract}

Keywords - Porous Titanium Oxide, Nanomaterials, Pattern Recognition, Fractal Dimension, Fractal Descriptors.

\section{Introduction}

Morphological material characterization is a very important and challenging task throughout Material Science, since such properties are determinant in the suitability of a given material to a specific application. Among several materials studied recently, a great deal attention has been given to 
photoactive ones, such as titanium oxide. Titanium oxide films prepared electrochemically can show a wide variation in their morphology depending on the experimental conditions in which they are prepared. As an example, the morphology can range from self-organized nanoporous [9, 19, 21] up to nanostructures without pores definition [3,5]. As described, many authors have shown that morphology is an important aspect to be considered in the photoactive properties of $\mathrm{TiO}_{2}$ films 3,13 . Recently we have shown that the photoactivity of $\mathrm{TiO}_{2}$ films prepared by galvanostatic anodization is strongly affected by the morphology on the early stages of the anodization process [18]. In [18] we have investigated the effect of morphology using an Image Analysis Method, which is based on the computational analysis of visual attributes, such as color, shape and texture. Among these attributes, texture is a powerful characterizer for material analysis. Although the concept of texture has no precise definition, it may be comprehended as the spatial organization of pixels in a digital image. A physical consequence of this definition is that this attribute is capable of express characteristics such as luminosity and roughness of a digitalized object. In this way, it allows a robust description and discrimination of material images, particularly $\mathrm{TiO}_{2}$ samples used in this study, which show few or no information about the pore diameter, but have a rich morphology related to texture information.

Unlike conventional textural data, natural textures do not present any evident quasi-periodic structure, but persistent random patterns [11]. Among the approaches employed for texture analysis, fractal methods are proposed as the best solution [12]. These methods, based on fractal dimension [14 or multifractal spectrum [10], can measure the complexity of an object texture, which corresponds to the shape irregularity, also related to the spatial occupation of an object. Therefore, fractal measurements are capable of quantifying the texture homogeneity, allowing a comparison among their information and the consequent discrimination of original materials. Using as example the materials here investigated, some authors [16, 20] have shown that the fractal dimension of $\mathrm{TiO}_{2}$ films can also be correlated to photoactivity properties of these materials. However, in that papers, the analysis is valid for very regular morphologies, showing deviations when more complex surfaces are considered.

Although the fractal dimension is a good descriptor to characterize a texture image, it is inefficient in applications involving the discrimination of a large amount of objects. In fact, it is easy to find objects with different aspects presenting the same fractal dimension [12]. In order to solve this drawback, the literature presents approaches which extract a lot of descriptors based on fractal geometry, such as Multifractals [10 and Multiscale 
Fractal Dimension (MFD) 4, 8, 15. In the literature, Backes et al. 11 applied MFD in texture analysis employing volumetric Bouligand-Minkowski MFD (VBMFD) to extract a set of descriptors from natural textures with very good results. VBMFD is based on the intensity image mapping onto a $3 \mathrm{D}$ surface. In the following, this surface is dilated by a variable radius $r$ and the volume $V(r)$ is calculated for each radius. This expansion process gives a precise measure of the pixel arrangement. As the radius $r$ grows, an interaction among dilation spheres is observed, interfering in $V(r)$ value. Therefore, these values capture changes in spatial distribution of textures along different scales. In this way, VBMFD uses $V(r)$ values as descriptors for a texture, capable of distinguishing different textures with their different spatial arrangement.

Considering the exposed above, in this work it is proposed the use of VBMFD descriptors to discriminate among nanoscale $\mathrm{TiO}_{2}$ films prepared electrochemically using two different experimental conditions and characterized by field emission gun scanning electron microscopy (FEGSEM) . In order to demonstrate the efficiency of the proposed method, this work obtains VBMFD descriptors from these films.

\section{Materials and Methods}

\subsection{Fractal Theory}

Since its introduction by Mandelbrot [14], the literature presents a lot of works using fractal theory to describe and discriminate several kinds of materials [16, 20]. Most of them use fractal dimension as a descriptor of the original samples. This is explained by the fact that fractal dimension measures the complexity of the object, related to the irregularity or the spatial occupation of that object. This property is strongly correlated to physical important properties.

\subsubsection{Fractal Dimension}

The fractal dimension 14 is the most commonly used measure to characterize a fractal object. Despite its importance, we cannot find a unique definition for this concept. The most ancient definition corresponds to the Hausdoff-Besicovitch dimension.

If $X \in \Re^{n}$ is a geometrical set of points, the Hausdoff-Besicovitch dimension $\operatorname{dim}_{H}(X)$ is given by:

$$
\operatorname{dim}_{H}(X)=\inf \left\{s: H^{s}(X)=0\right\}=\sup \left\{H^{s}(X)=\infty\right\},
$$


where $H^{s}(X)$ is the $s$-dimensional Hausdorff measure, given by:

$$
H^{s}(X)=\liminf _{\delta \rightarrow 0}\left\{\sum_{i=1}^{\infty}\left|U_{i}\right|^{s}: U_{i} \text { is a } \delta \text {-cover of } \mathrm{X}\right\},
$$

where $\|$ expresses a diameter in $\Re^{n}$, that is, $|U|=\sup |x-y|: x, y \in U$.

Otherwise, the Hausdorff-Besicovitch definition application could be impracticable in many real situations. This is the case of discrete objects represented in a digital image, as those studied here. For such applications, we have an alternative definition of fractal dimension, which is a generalization from the topological dimension. Thus, the fractal dimension $D$ is provided by:

$$
D(X)=\lim _{\epsilon \rightarrow 0} \frac{\log (N(\epsilon))}{\log \left(\frac{1}{\epsilon}\right)},
$$

where $N(\epsilon)$ is the number of objects with linear size $\epsilon$ required to cover the whole object $X$ [7. More generically, $N(\epsilon)$ may be considered a measure which varies according to the scale $\epsilon$. Such measure is characterized by a power-law relation with the scale [17]. A lot of fractal dimension methods were developed by using distinct measures, such as Bouligand-Minkowski [7], box-counting [7], Fourier [17]. Here, we are focused on the BouligandMinkowski fractal dimension.

\subsubsection{Bouligand-Minkowski}

The original definition of Bouligand-Minkowski fractal dimension $\operatorname{dim}_{B}(X)$ depends on a symmetrical structuring element $Y$ :

$$
\operatorname{dim}_{M}(X, Y)=\inf \left\{\tau, \text { meas }_{M}(X, Y, \tau)=0\right\},
$$

where meas $_{M}$ is the Bouligand-Minkowski measure:

$$
\operatorname{meas}_{M}(X, Y, \tau)=\lim _{r \rightarrow 0} \frac{V(\partial X \oplus r Y)}{r^{n-\tau}},
$$

where $r$ is the radius of $Y$ and $V$ is the volume of the dilation between $Y$ and the boundary $\partial X$ of $X$.

By using neighborhood techniques we may find a simplified version of this dimension, which eliminates the explicit dependence from $Y$ :

$$
\operatorname{dim}_{M}(X)=\lim _{\epsilon \rightarrow 0}\left(D_{T}-\frac{\log V\left(X \oplus Y_{\epsilon}\right)}{\log \epsilon}\right) .
$$


For the cases such as those studied here, when $X \in \Re^{3}$, the topological dimension is $D_{T}=3$ and $Y_{\epsilon}$ is a sphere with diameter $\epsilon$. For each value of $\epsilon$, each point in $X$ is dilated by $Y_{\epsilon}$ and the number of points inside the dilated structure is the dilation volume $V$.

\subsection{Proposed Method}

A significative drawback of fractal dimension is that it is only a unique real number. Therefore, it is possible to find a great number of objects which despite its same fractal dimension present appearance completely diverse. This is exemplified in the Figure 1 using textures from Brodatz data set [2]. The authors in 15] proposed the Multiscale Fractal Dimension technique to solve this issue. In MFD, the fractal dimension is calculated for the object observed under different spatial scales and each value is used as a descriptor for the object. In this work, we propose the analysis of nanoscale FEG images by applying a multiscale approach to the Bouligand-Minkowski fractal dimension.
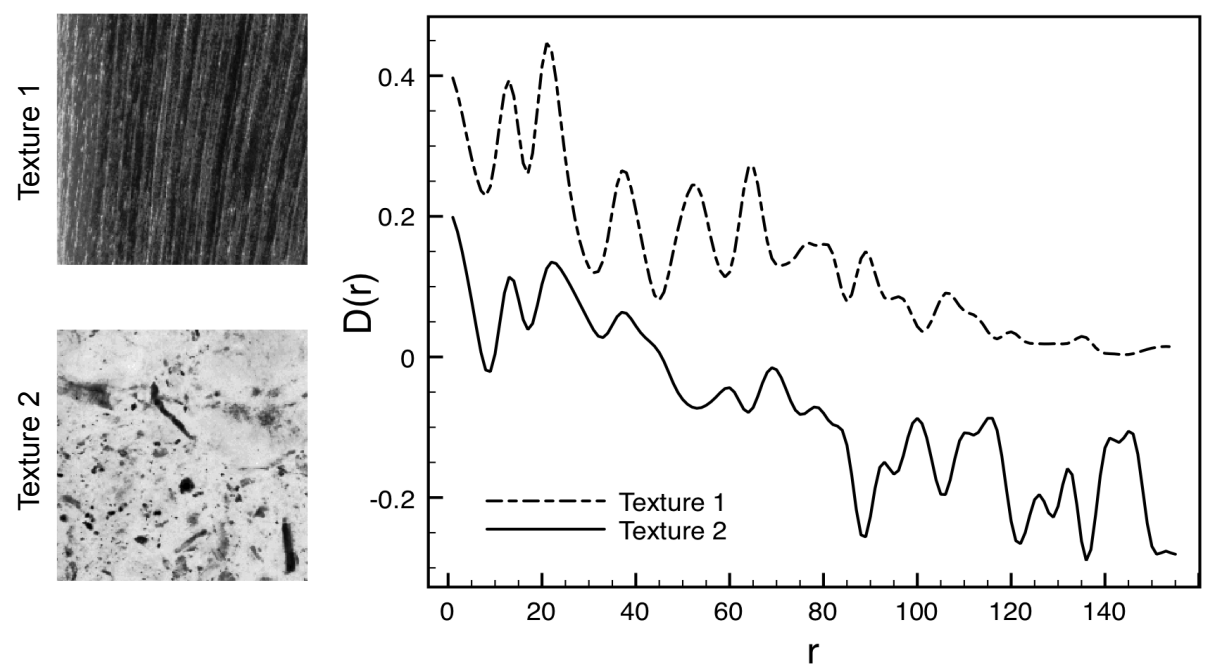

Figure 1: Two textures whose surfaces present the same fractal dimension $(\mathrm{FD}=2.014)$ and their multiscale fractal descriptors $D(r)$, where $r$ is the descriptor index.

Initially, we map the image $\operatorname{Img} \in[1: M] \times[1: N] \rightarrow \Re$ onto a 
threedimensional surface

$$
\text { Surf }=\{i, j, f(i, j) \mid(i, j) \in[1: M] \times[1: N]\},
$$

such that:

$$
f(i, j)=\left\{1,2, \ldots, \text { max }_{-} g r a y\right\} \mid f=\operatorname{Im} g(i, j),
$$

where max_gray is the maximum pixel intensity.

In the following, the surface is dilated by a variable radius $r$, as depicted in Figure 4. Then the dilation volume $V(r)$ for each dilation radius is calculated. The value of $V(r)$ also corresponds to the number of points with a distance at most $r$ from the object. Therefore, the exact Euclidean distance transform (EDT) 6] becomes a powerful and efficient tool for this calculus.

In the threedimensional space, EDT may be defined as the distance of each point in the space to a subset of it. In our case, this subset is the surface and the EDT for each point outside Surf is given by:

$$
\operatorname{EDT}(p)=\min \left\{d(p, q) \mid q \in \operatorname{Surf}^{c}\right\},
$$

where $d$ is the Euclidean distance.

In particular, in exact EDT, the distances present discrete values $E$ :

$$
E=0,1, \sqrt{2}, \ldots, l, \ldots
$$

where

$$
l \in D=\left\{d \mid d=\left(i^{2}+j^{2}\right)^{1 / 2} ; i, j \in \mathbb{N}\right\}
$$

The dilation volume is given through:

$$
V(r)=\sum_{i=1}^{r} Q(i),
$$

where

$$
Q(r)=(x, y, z) \mid g_{k}(P)-\left[g_{r}(P) \cap \cup_{i=0}^{r-1} g_{i}(P)\right],
$$

such that:

$$
g_{r}(P)=\left\{(x, y, z) \mid\left[\left(x-P_{x}\right)^{2}+\left(y-P_{y}\right)^{2}+\left(z-P_{z}\right)^{2}\right\},\right.
$$

where

$$
P=(x, y, z) \mid f(x, y, z) \in \operatorname{Surf}
$$

The authors in [1] propose the use of values $V(r)$ as descriptors for texture. Such technique is named Volumetric Bouligand-Minkowski Multiscale 
Fractal Dimension (VBMFD). In that work, the authors apply this technique to the analysis of plant leaves, achieving interesting results. It is important to stress out that $V(r)$ is directly related to the Bouligand-Minkowski dimension for maximum radius $r$. Besides, each radius corresponds to an observation scale, from further (greater radius) to closer (smaller radius).

This work proposes the application of VBMFD descriptors for the analysis of two $\mathrm{TiO}_{2}$ samples prepared under different experimental conditions. The goal is to discriminate between the samples based on images extracted from different regions of each film.

\subsection{Sample Preparation}

The samples were prepared as follows. Titanium sheet (Alfa Aesar, 99.99\%, $0.25 \mathrm{~mm}$ thick) with an exposed area of $1 \mathrm{~cm}^{2}$, and two platinum sheets were used as working and counter electrodes, respectively. Before the anodization process, the working electrode was polished with \#1000 $\mathrm{SiC}$ and then with \#1200 SiC emery paper followed by vigorous washing with deionized water.

The experiments were carried out under galvanostatic conditions with a home-made current source, measuring the potential difference between working electrode and counter electrode with an HP 34410A multimeter coupled to a computer by an in-house software routine developed with HPVEE 5.0 software. The two samples investigated were prepared in oxalic acid (sample 1 in a concentration of $0.05 \mathrm{~mol} \mathrm{~L}^{-1}$, and sample 2 in $0.5 \mathrm{~mol} \mathrm{L^{-1 }}$ ) applying a constant current density of $10 \mathrm{~mA} \mathrm{~cm}^{-2}$ and $20 \mathrm{~mA} \mathrm{~cm}^{-2}$ (sample 1 and sample 2 , respectively) on the working electrode. The temperature was kept constant at $10{ }^{\circ} \mathrm{C}$ during the anodization of both samples.

After the preparation, the samples were morphlogically characterized using a field emission gun scanning electron microscope (Supra35 - Zeiss). From both samples we have extracted ten images from different regions of the material in order to collect a representative amount of data.

\section{Results}

The fractal descriptors were used for the discrimination of two $\mathrm{TiO}_{2}$ films prepared under different experimental conditions. From each sample, we have extracted two micrographs, represented in the Figure 2.

Initially, we apply the transform which maps the intensity image onto a surface, as illustrated in the Figure 3.

Next, the surface is dilated by a variable radius $r$, as depicted in the Figure 4. The conventional Bouligand-Minkowski dimension technique is 

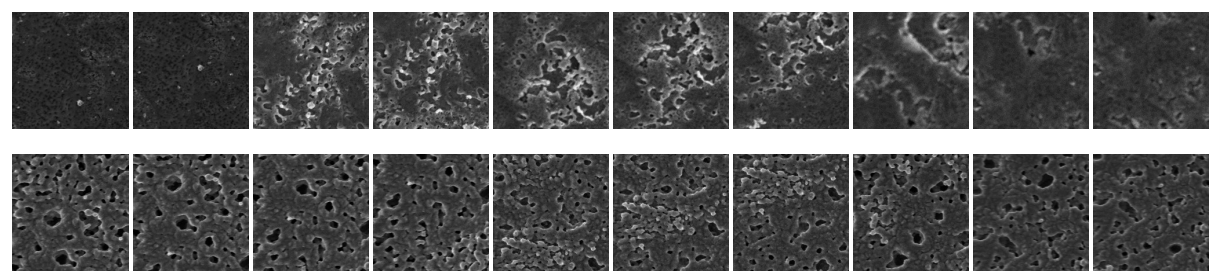

Figure 2: Texture images extracted from titanium oxide under two different conditions (above and below).
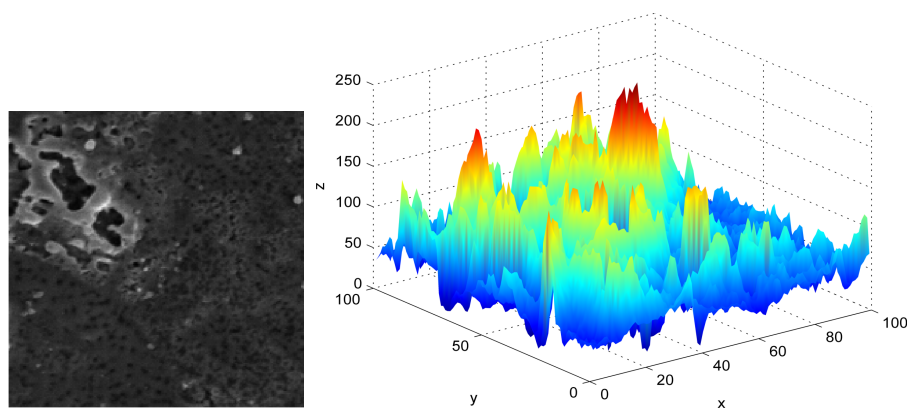

Figure 3: Texture mapped onto a surface. (a) Original texture. (b) Threedimensional surface.

extracted from the curve $\log (V(r)) \times r$, in which, $V(r)$ is the diation volume. Here, the descriptors obtained by the VBMFD technique are provided by:

$$
D^{\prime}(r)=\log (V(r))
$$

In order to eliminate redundancies from descriptors $D_{k}^{\prime}(r)$ of the $k^{\text {th }}$ image the following operation was performed:

$$
D_{k}(r)=D_{k}^{\prime}(r)-\sum_{i=1}^{n} D_{i}^{\prime}(r) / n,
$$

where $n$ is the total number of images in the dataset and $D_{k}(r)$ is the used descriptor.

Figure 5 shows the descriptor curves for each image from $\mathrm{TiO}_{2}$ samples. Each line aspect (solid and dashed) corresponds to a sample prepared in a different experimental condition. 

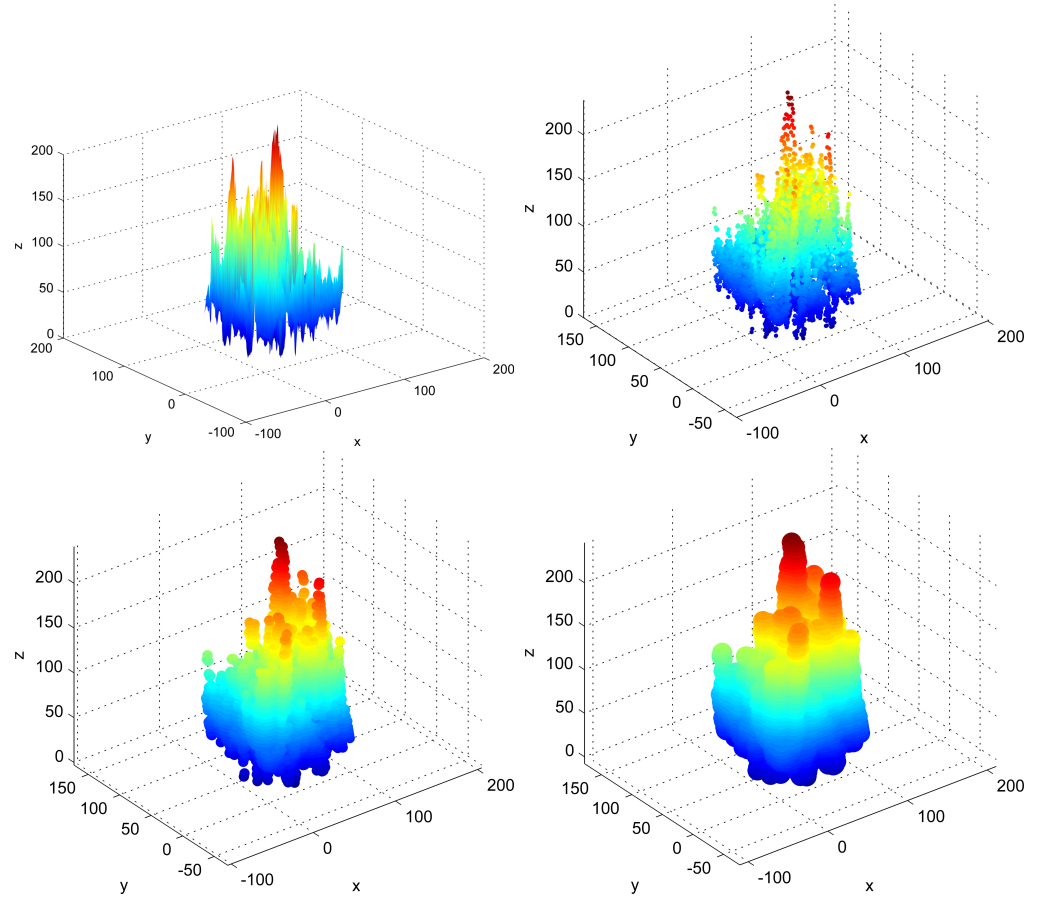

Figure 4: Dilated surfaces with different radii. (a) Original surface. (b) Radius 2. (c) Radius 5. (d) Radius 10.

It is noticeable that descriptors curve aspects discriminate strongly the materials. We do not observe any interlacement among curves from different conditions. The whole graphic reflects a high inter-class variability and a low intra-class variation in descriptors. Such result is in accordance to the pixel arrangement nature, measured through fractal descriptors.

Moreover, we notice the complex shape of each curve, reflecting the richness of information expressed in the descriptors. We observe a clear improve in the description compared to the classical fractal dimension.

Figure 6 shows the distribution of two statistical important scores of fractal descriptors: from Principal Component Analysis (PCA) and Canonical Correlation Analysis (CCA).

While PCA plot measures the dispersion of whole dataset, CCA measures the correlation among data from a same material. Both graphics show a cluster of points (corresponding to the 2 main scores) in each material conditions, comprobing again the discrimination power of fractal descriptors. 


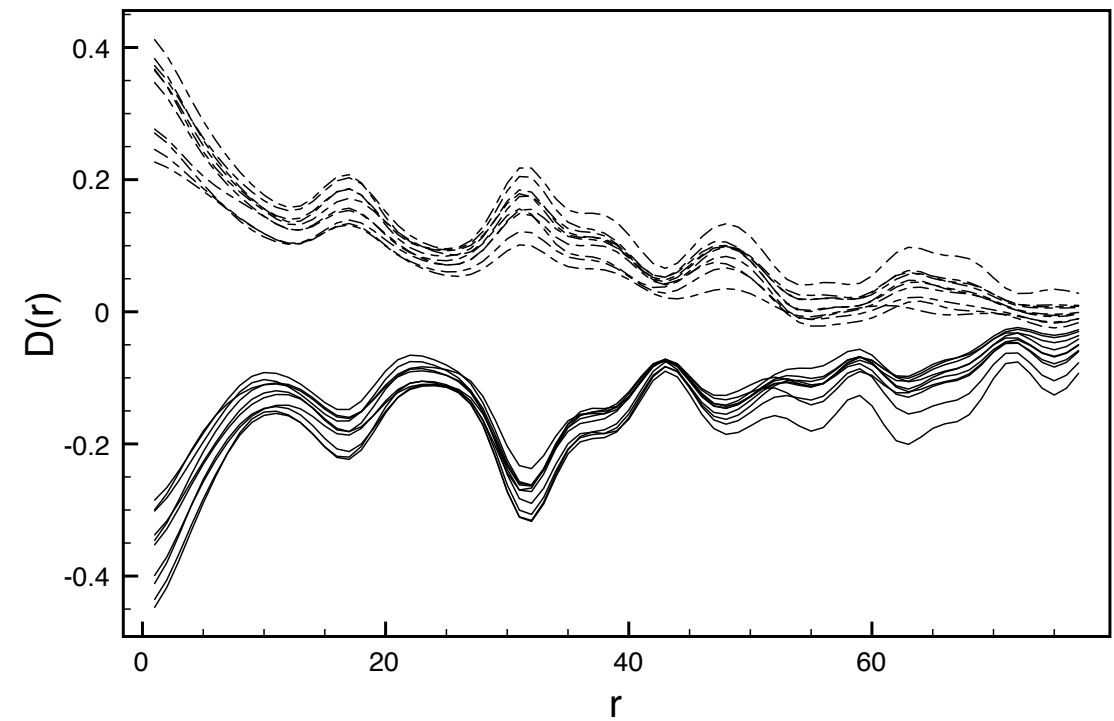

Figure 5: Descriptors curves from each texture image. Solid curves correspond to sample 1. Dashed ones correspond to sample 2.
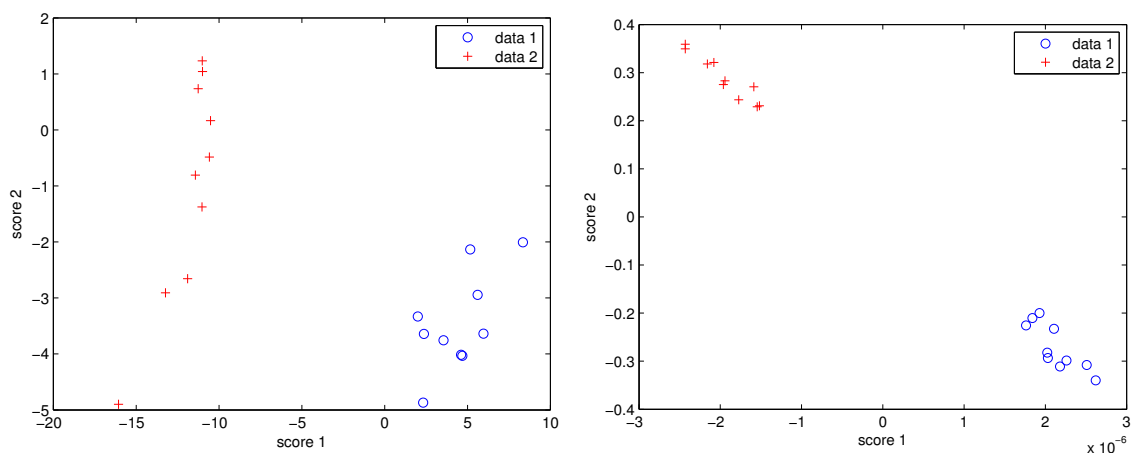

Figure 6: Distribution of correlation scores. (a) Principal Component Analysis. (b) Canonical Correlation Analysis.

\section{Conclusions}

In this work, we proposed the application of fractal descriptors to the discrimination of materials under different experimental conditions. We obtained the descriptors by applying a multiscale approach to the fractal dimension estimation of texture images extracted from the material. 
The curves of descriptors showed a high accuracy in the discrimination. This result demonstrated the power of the proposed technique in the modelling of materials. It also comprobed the validity of materials analysis through computational texture analysis.

\section{References}

[1] Backes, A.R., Casanova, D., Bruno, O.M.: Plant leaf identification based on volumetric fractal dimension. International Journal of Pattern Recognition and Artificial Intelligence (IJPRAI) 23(6), 1145-1160 (2009)

[2] Brodatz, P.: Textures: A photographic album for artists and designers. Dover Publications, New York (1966)

[3] Brunella, M., Diamanti, M., Pedeferri, M., Fonzo, F.D., Casari, C., Bassi, A.L.: Photocatalytic behavior of different titanium dioxide layers. Thin Solid Films 515(16), 6309-6313 (2007)

[4] Bruno, O.M., de Oliveira Plotze, R., Falvo, M., de Castro, M.: Fractal dimension applied to plant identification. Inf. Sci 178(12), 2722-2733 (2008)

[5] Cai, Q., Yang, L., Yu, Y.: Investigations on the self-organized growth of tio2 nanotube arrays by anodic oxidization. Thin Solid Films 515(4), 1802-1806 (2006)

[6] Fabbri, R., Costa, L.D.F., Torelli, J.C., Bruno, O.M.: 2d euclidean distance transform algorithms: A comparative survey. ACM Comput. Surv. 40(1), 1-44 (2008)

[7] Falconer, K.J.: The Geometry of Fractal Sets. Cambridge University Press, Cambridge, UK (1985)

[8] Florindo, J.B., De Castro, M., Bruno, O.M.: Enhancing Multiscale Fractal Descriptors Using Functional Data Analysis. International Journal of Bifurcation and Chaos 20(11), 3443-3460 (2010)

[9] Ghicov, A., Tsuchiya, H., Macak, J.M., Schmuki, P.: Titanium oxide nanotubes prepared in phosphate electrolytes. Electrochemistry Communications 7(5), 505-509 (2005) 
[10] Harte, D.: Multifractals: theory and applications. Chapman and Hall/CRC (2001)

[11] Huang, P., Dai, S., Lin, P.: Texture image retrieval and image segmentation using composite sub-band gradient vectors. Journal of Communication and Image Representation 17(5), 947-957 (2006)

[12] Kaplan, L.: Extended fractal analysis for texture classification and segmentation. IEEE Transactions on Image Processing 8(11), 1572-1585 (1999)

[13] Mac·k, J.M., Tsuchiya, H., Ghicov, A., Schmuki, P.: Dye-sensitized anodic tio2 nanotubes. Electrochemistry Communications 7(11), 1133$1137(2005)$

[14] Mandelbrot, B.B.: The Fractal Geometry of Nature. Freeman (1968)

[15] Manoel, E.T.M., da Fontoura Costa, L., Streicher, J., Müller, G.B.: Multiscale fractal characterization of three-dimensional gene expression data. In: SIBGRAPI, pp. 269-274. IEEE Computer Society (2002)

[16] Provata, A., Falaras, P., Xagas, A.: Fractal features of titanium oxide surfaces. Chemical Physics Letters 297(5-6), 484-490 (1998)

[17] Russ, J.C.: Fractal Surfaces. Plenum Press, New York (1994)

[18] Sikora, M.d.S., Rosario, A.V., Pereira, E.C., Paiva-Santos, C.O.: Influence of the morphology and microstructure on the photocatalytic properties of titanium oxide films obtained by sparking anodization in h3po4. Electrochimica Acta 56(9), 3122-3127 (2011)

[19] Tsuchiya, H., Macak, J.M., Taveira, L., Balaur, E., Ghicov, A., Sirotna, K., Schmuki, P.: Self-organized tio2 nanotubes prepared in ammonium fluoride containing acetic acid electrolytes. Electrochemistry Communications $\mathbf{7}(6), 576-580$ (2005)

[20] Xagas, A.P., Androulaki, E., Hiskia, A., Falaras, P.: Preparation, fractal surface morphology and photocatalytic properties of tio2 films. Thin Solid Films 357(2), 173-178 (1999)

[21] Zlamal, M., Macak, J.M., Schmuki, P., Kr"sa, J.: Electrochemically assisted photocatalysis on self-organized tio2 nanotubes. Electrochemistry Communications 9(12), 2822-2826 (2007) 